TITLE:

\title{
Highly localized photothermal conversion in two-dimensional Au nanoparticle arrays
}

$\operatorname{AUTHOR}(S):$

Namura, Kyoko; Suzuki, Motofumi; Nakajima, Kaoru; Kimura, Kenji

\section{CITATION:}

Namura, Kyoko ... [et al]. Highly localized photothermal conversion in two-dimensional Au nanoparticle arrays. Journal of Applied Physics 2013, 114(7): 074308.

\section{ISSUE DATE:}

2013

URL:

http://hdl.handle.net/2433/192289

\section{RIGHT:}

Copyright 2013 American Institute of Physics. This article may be downloaded for personal use only. Any other use requires prior permission of the author and the American Institute of Physics. 


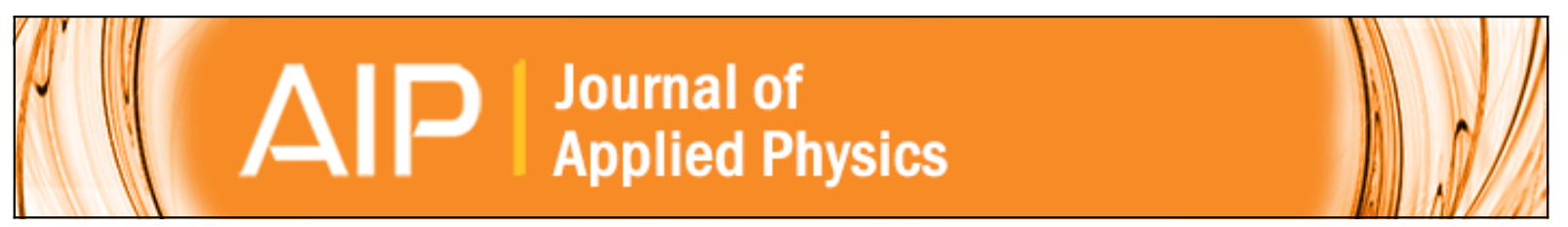

\section{Highly localized photothermal conversion in two-dimensional Au nanoparticle arrays}

Kyoko Namura, Motofumi Suzuki, Kaoru Nakajima, and Kenji Kimura

Citation: Journal of Applied Physics 114, 074308 (2013); doi: 10.1063/1.4818814

View online: http://dx.doi.org/10.1063/1.4818814

View Table of Contents: http://scitation.aip.org/content/aip/journal/jap/114/7?ver=pdfcov

Published by the AIP Publishing

\section{Articles you may be interested in}

Enhanced and polarization dependence of surface-enhanced Raman scattering in silver nanoparticle arraynanowire systems

Appl. Phys. Lett. 102, 163108 (2013); 10.1063/1.4802802

Chemical coating of large-area Au nanoparticle two-dimensional arrays as plasmon-resonant optics

Appl. Phys. Lett. 97, 221101 (2010); 10.1063/1.3518469

Image dipoles approach to the local field enhancement in nanostructured Ag-Au hybrid devices

J. Chem. Phys. 132, 024712 (2010); 10.1063/1.3291438

Optical generation of high frequency ultrasound using two-dimensional gold nanostructure

Appl. Phys. Lett. 89, 093901 (2006); 10.1063/1.2344929

Fabrication and near-infrared photothermal conversion characteristics of Au nanoshells

Appl. Phys. Lett. 86, 113109 (2005); 10.1063/1.1874308

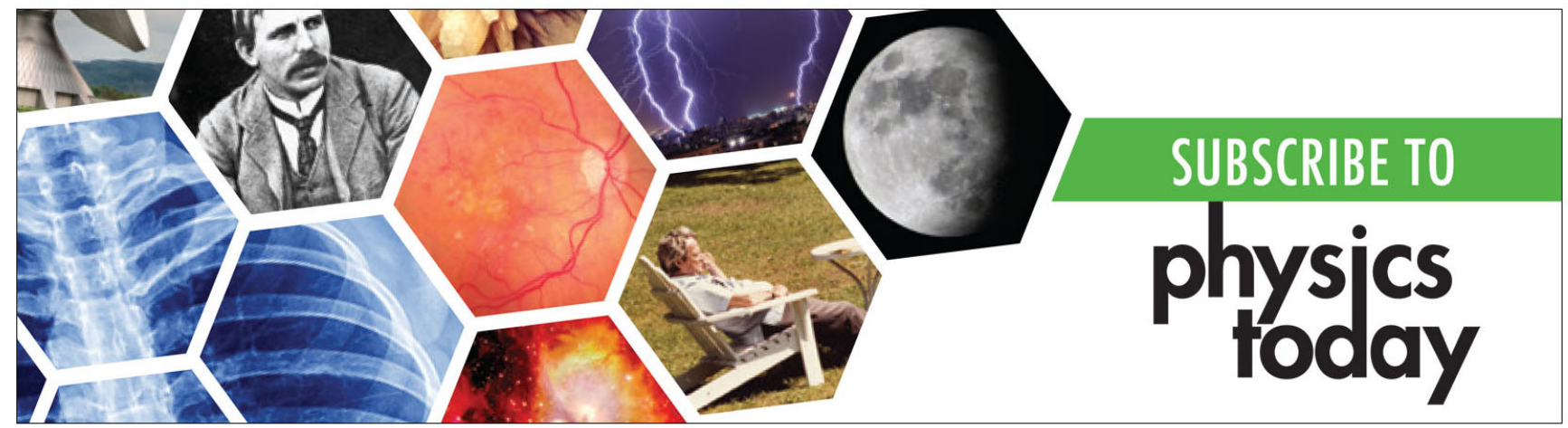




\title{
Highly localized photothermal conversion in two-dimensional Au nanoparticle arrays
}

\author{
Kyoko Namura, a),b) Motofumi Suzuki, Kaoru Nakajima, and Kenji Kimura \\ Nanomaterials Eng. Lab., Department of Micro Engineering, Kyoto University-Katsura, \\ Kyoto 615-8540, Japan
}

(Received 6 June 2013; accepted 2 August 2013; published online 19 August 2013)

\begin{abstract}
We have investigated, both theoretically and experimentally, highly localized photothermal conversions in Au nanoparticle array/dielectric layer/Ag mirror sandwiches, namely local plasmon resonators. The depth profile of the optical absorption in the local plasmon resonators was calculated using a simple model comprising homogeneous multilayers. The calculation results show highly localized light absorption in the $\sim 10$-nm-thick Au nanoparticles layer (more than 99\% of total optical absorption). The photoacoustic measurements, which are sensitive to the surface temperature of the sample, were performed on the fabricated local plasmon resonators. The photoacoustic amplitude of the local plasmon resonator possessing a high optical absorption $(A=0.97)$ was 15 times larger than the absorbance of the bulk $\mathrm{Si}$ wafer $(A=0.67)$ and 8 times larger than the absorbance of graphite $(A=0.85)$. These results suggest that the photothermal conversion is localized in the thin Au nanoparticles layer, which enables rapid modulation of the temperature of the surrounding fluid. (C) 2013 AIP Publishing LLC. [http://dx.doi.org/10.1063/1.4818814]
\end{abstract}

\section{INTRODUCTION}

The photothermal effect in noble metal nanoparticles (NPs) is critical to various applications, including biomedicine, ${ }^{1,2}$ chemical catalysis, ${ }^{3}$ energy ${ }^{4,5}$ microfluidics, ${ }^{6-8}$ and photoacoustic effects, ${ }^{9,10}$ because they are capable of acting as localized heat sources at nanometer scale. This effect is based on the plasmon resonances in the noble metal NPs, and there have been numerous attempts to maximize their optical absorption and optimize their resonance wavelength by manipulating their size and shape ${ }^{11,12}$ or by combining them with dielectric materials. ${ }^{13}$ Recently, it was demonstrated that $\mathrm{Au}$ NPs/dielectric layer/Ag mirror sandwich structures, namely the local plasmon resonators, realize a wide range of optical absorption from 0.03 to 0.97 because of the strong interference. ${ }^{14}$ These sandwich structures can be self-assembled by dynamic oblique deposition (DOD). In our previous study, ${ }^{14}$ we demonstrated a large temperature increase in water by photothermal conversion utilizing a sample with high optical absorption and showed that heat generation is proportional to optical absorption. These properties make the local plasmon resonators attractive as efficient nano-heaters. However, we have not addressed the optical absorption by each layer of the sandwich structure. These materials contain two layers that possess high extinction coefficients: the Au NPs layer and the Ag mirror layer. It is important to elucidate the contribution of these layers to photothermal conversion in order to analyze and control the local temperature at nanometer scale. In this study, we quantitatively determine the highly localized light absorption of the Au NPs layer. Additionally, we experimentally demonstrated efficient photoacoustic emission resulted in the

\footnotetext{
${ }^{\text {a)} E l e c t r o n i c ~ m a i l: ~ n a m u r a . k y o k o .57 r @ s t . k y o t o-u . a c . j p . ~}$

${ }^{b)}$ Research Fellow of the Japan Society for the Promotion of Science.
}

photothermal conversion of the thin Au NPs layer, which was approximately $10 \mathrm{~nm}$ thick.

\section{OPTICAL MODEL}

First, we describe the optical properties of the local plasmon resonator which consists of $\mathrm{Au} \mathrm{NPs} / \mathrm{SiO}_{2}$ shape control layer (SCL) $/ \mathrm{SiO}_{2}$ phase control layer (PCL)/Ag mirror layer by using a simple model (Fig. 1). Figures 1(a) and 1(b) show the typical SEM images of the local plasmon resonator. ${ }^{14}$ Because the dimensions of the nanostructures in the various layers are much smaller than the wavelengths in the nearinfrared or other longer wavelength regions, we treated our thin films as homogeneous multilayers (Fig. 1(c)). The thicknesses of the layers have been defined in Fig. 1(c) and the thicknesses of SCL, PCL, and Ag layers are determined from the SEM images. Since we vary the PCL thicknesses in the model, we represent their values by $d_{p c l}$. For the Au NPs layer, $\mathrm{Au}$ is deposited to an average thickness of $10 \mathrm{~nm}$. Because the Au NPs layer forms an island structure, its geometrical thickness is defined as $10 / f \mathrm{~nm}$ where $f$ is the volume fraction of Au. The refractive index of each layer is defined as follows. The Au NPs layer is treated as an effective medium of a mixture of a metal nano-sphere and a transparent material having the same dielectric constant as the SCL. The effective dielectric function is evaluated by the Maxwell-Garnett model $^{15}$ as

$$
\epsilon^{a v}=\epsilon_{s c l} \frac{(1+2 f) \epsilon+2(1-f) \epsilon_{s c l}}{(1-f) \epsilon+(2+f) \epsilon_{s c l}} .
$$

Here, $f$ is the volume fraction of the nano-spheres, $\epsilon$ is the dielectric function of the metal, and $\epsilon_{s c l}$ is the in-plane dielectric function of the SCL, which will be defined later. From the SEM observations, $f$ is estimated to be 


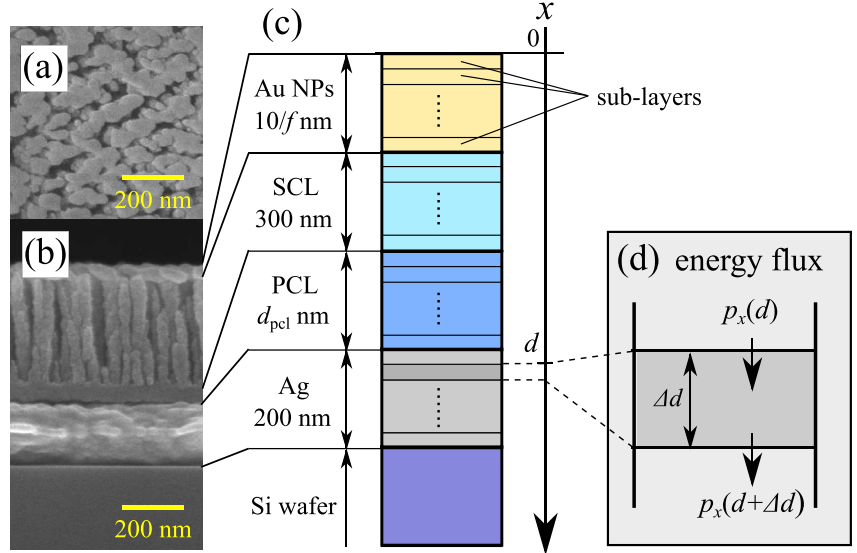

FIG. 1. Modeling of local plasmon resonators whose structures are shown in the SEM images ((a) surface morphology and (b) cross section). They are described by (c) multilayered homogeneous structures that have Au NPs/ SCL/PCL/Ag layers. Each layer is divided into sublayers in order to calculate optical absorption distribution. Figure (d) shows energy balance within a single sublayer.

$0.5 \leq f \leq 1$. The dielectric function of the metal, $\epsilon$, for the incident photon energy $\omega$, is represented by the Drude model

$$
\epsilon=\epsilon_{\infty}-\frac{\omega_{p}{ }^{2}}{\omega(\omega+i \delta)},
$$

where $\omega_{p}$ is the plasma frequency, $\epsilon_{\infty}$ is the dielectric constant at high frequencies, and $\delta$ is the reciprocal of the scattering time. The bulk values of $\omega_{p}=8.96 \mathrm{eV}, \epsilon_{\infty}=7.44$ are standard values, ${ }^{16,17}$ and $\delta$ is used as the fitting parameter, because corresponding value for nano-structures could be larger compared to the bulk value. For the SCL, we assumed in-plane refractive index $\sqrt{\epsilon_{s c l}}=n_{s c l}$ for normal incident light. Because the packing density of an SCL is small, its inplane refractive index is estimated to be smaller than that of the bulk $\mathrm{SiO}_{2}$, so that $1 \leq n_{s c l} \leq 1.47$. The PCL consists of $\mathrm{SiO}_{2}$ that has a refractive index of $n_{p c l}=1.47$. The refractive index of the Ag mirror layer is reported in the literature. ${ }^{18}$

The optical absorption spectra were calculated via a transfer matrix formalism ${ }^{15}$ at normal incidence with unpolarized light and fitted to the experimental results using fitting parameters $f, \delta$, and $n_{s c l}$ (Fig. 2). Because the optical transmittance and scattering of our samples are negligibly small, the optical absorption $A$ is evaluated as $A=1-R$, where $R$ is the optical reflectance. The value of $n_{s c l}$ determines the cycle of the periodic undulation observed in the optical absorption spectra, which arises from interference. Thus, $n_{s c l}$ was chosen to fit the peak positions of the undulation of the calculated spectra to the positions of the measured spectra. On the other hand, the peak shape and intensity are sensitive to both $\delta$ and $f$ of the Au NPs layer. Therefore, $n_{s c l}$, $\delta$, and $f$ were chosen to reproduce the intensity and shape of the absorption peaks. Figure 2 shows the fitting results, which agree well with experimental results when $\delta=1.8$, $f=0.75$, and $n_{s c l}=1.25$. The fitting results of $f$ and $n_{s c l}$ are consistent with the SEM images. The value of $\delta$ is significantly larger than the bulk value, because the scattering probability increases as a result of the defects and surface roughness of NPs. The calculation results agree well with the

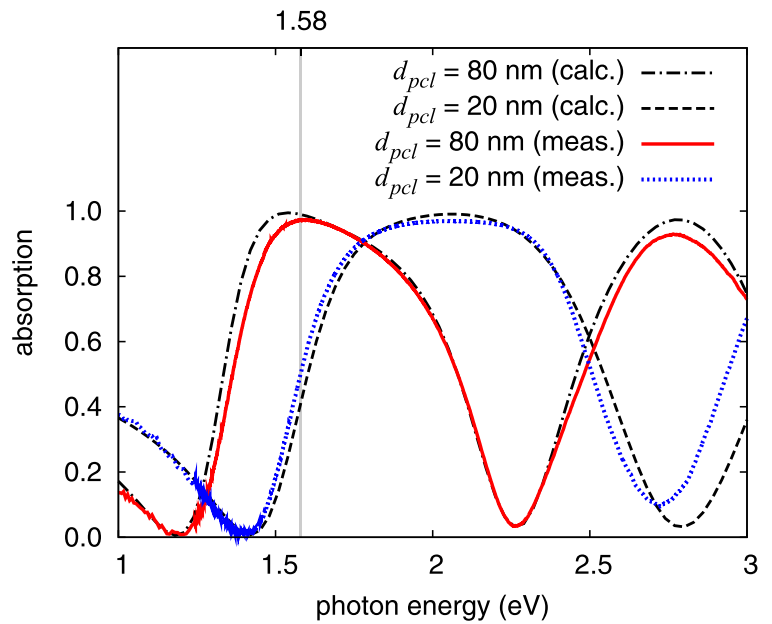

FIG. 2. Calculated optical absorption spectra of local plasmon resonators with $d_{p c l}=20$ and $80 \mathrm{~nm}$.

experimental results for both the values of $d_{p c l}$, i.e., 20 and $80 \mathrm{~nm}$. Because this model reflects the optical characteristics of our samples quite well, we utilized it to discuss the optical absorption of each layer.

Each layer of the model structure was divided into thin sub-layers of $\Delta d$ thickness (Fig. 1(c)), and the optical absorption for each sub-layer was calculated by utilizing the Poynting vector, $p_{x}$, which represents energy flux along the $x$ axis and is given by the following equation:

$$
p_{x}=\frac{1}{2} \operatorname{Re}\left\{E_{y} H^{*}{ }_{z}-E_{z} H^{*}{ }_{y}\right\},
$$

where $E_{y}, H_{y}, E_{z}, H_{z}$ are in-plane electromagnetic fields at the position $x$, and the subscripts " $y$ " and " $z$ " denote the $y$ and $z$ components. Regarding the sub-layer at the position $x=d$ (Fig. 1(d)), the optical absorption per unit length, $\alpha_{e f f}$, can be deduced from following equation:

$$
\alpha_{e f f}(d)=\frac{p_{x}(d)-p_{x}(d+\Delta d)}{\Delta d I_{0}},
$$

where $I_{0}$ is the intensity of the incident light. We name $\alpha_{e f f}$ as "effective absorption coefficient," because its integration over the $x$ axis gives the total absorption, $A$, of the sample.

Figure 3 shows the calculation results of the effective absorption coefficient at the wavelength of $785 \mathrm{~nm}(1.58 \mathrm{eV})$ as a function of the position from the surface. The effective absorption coefficient of the Au NPs layer of the local plasmon resonator $(A=0.99)$ is three orders of magnitude higher than that of the $\mathrm{Ag}$ mirror layer. Thus, the optical absorption of the local plasmon resonator is localized in the $\sim 10-\mathrm{nm}$ thick Au NPs layer, which lies at the sample surface. On the other hand, the effective absorption coefficient of the Si wafer is three orders of magnitude smaller than that of the Au NPs layer in the local plasmon resonator and nearly at a depth of $500 \mathrm{~nm}$ from the surface. It decreases gradually over several micrometers (Fig. 3(b) inset). This result suggests that the $\mathrm{Si}$ wafer has an absorption layer that is several micrometers thick. Thus, local plasmon resonators realize highly localized light absorption compared to bulk absorbing materials. 

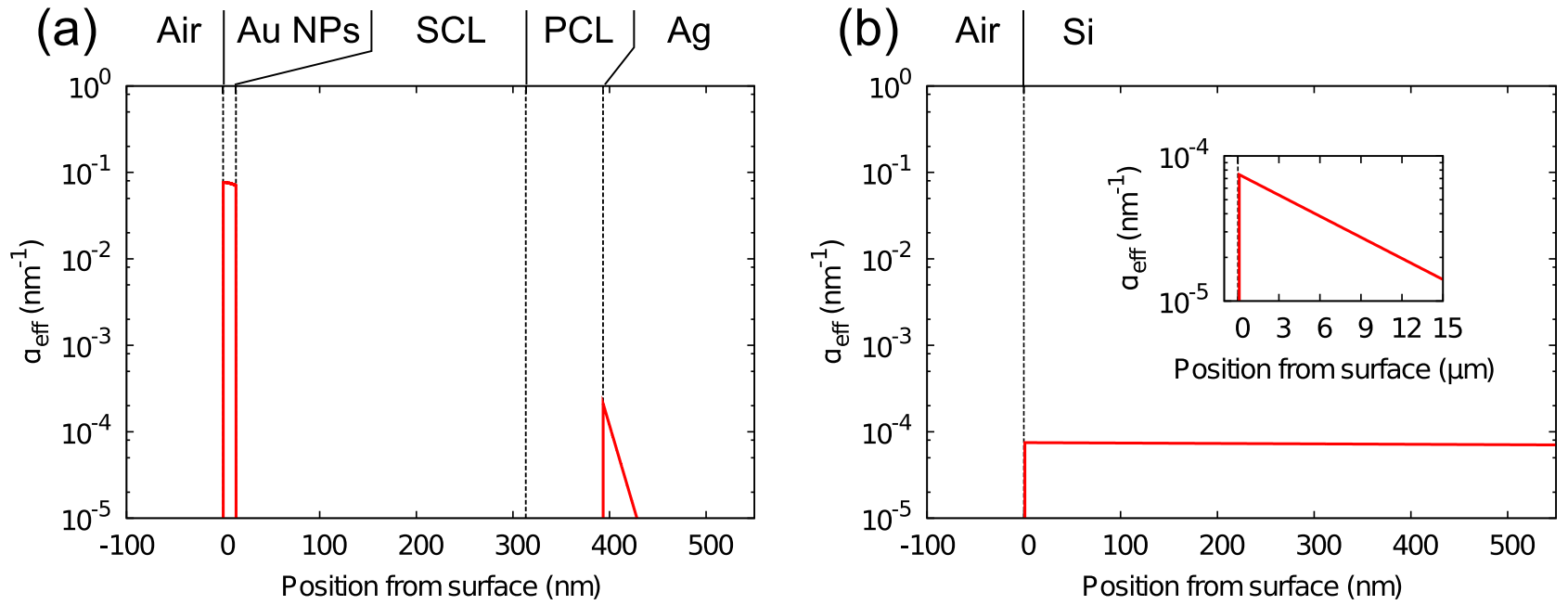

FIG. 3. Effective absorption coefficient, $\alpha_{e f f}\left(\mathrm{~nm}^{-1}\right)$, as a function of position from surface for (a) local plasmon resonator with 80 -nm-thick PCL ( $A=0.99$ ) and (b) $\mathrm{Si}$ wafer $(A=0.67)$ at the wavelength of $785 \mathrm{~nm}$. Inset in (b) shows a gradual decrease in $\alpha_{e f f}$ over several micrometers in the Si wafer.

For quantitative analysis, the optical absorption of each layer of the local plasmon resonators was calculated for the samples with $d_{p c l}=20,80$, and $250 \mathrm{~nm}$ (Table I). It is important to note that the calculated total absorption of these samples varies from $\sim 0.01$ to 0.99 , which suggests that the photothermal conversion of the local plasmon resonators can be spatially controlled through the patterning of $d_{p c l}$. Regardless of the variation of the total absorption, the absorption of the $\mathrm{Ag}$ mirror layer is negligibly small. In this calculation, up to $99.6 \%$ of the total absorption is absorbed by the Au NPs layer when the total optical absorption is high $(A=0.99)$. This result suggests that the local plasmon resonators can efficiently convert light energy to heat energy in the AuNPs layer and can drastically alter the surface temperature. Therefore, we focused our attention on the photoacoustic effect which is known to be sensitive to the sample surface temperature modulation ${ }^{19}$ and performed photoacoustic measurements on the fabricated local plasmon resonators.

\section{EXPERIMENTS}

The local plasmon resonators were self-assembled by DOD. The preparation method of the Au NPs layer is essentially the same as that reported in a previous publication. ${ }^{20}$ First, a 200-nm-thick smooth Ag layer and a PCL of $\mathrm{SiO}_{2}$ with $d_{p c l}=20$ or $80 \mathrm{~nm}$ were deposited on a Si wafer. On the PCL, a 300-nm-thick SCL of $\mathrm{SiO}_{2}$ was prepared. During the deposition of the SCL, the deposition angle measured from the surface normal was fixed at $80^{\circ}$, and the substrate was

TABLE I. Calculated optical absorption for each layer of local plasmon resonators with $d_{p c l}=20,80$, and $250 \mathrm{~nm}$ at the wavelength of $785 \mathrm{~nm}$.

\begin{tabular}{lcccccc}
\hline \hline & \multirow{2}{*}{$d_{p c l}(\mathrm{~nm})$} & Total absorption & & \multicolumn{4}{c}{ Absorption for each layer } \\
\cline { 2 - 3 } \cline { 5 - 7 } & $A$ & & Au NPs & SCL & PCL & Ag \\
\hline 20 & 0.404 & & 0.339 & 0.000 & 0.000 & 0.005 \\
80 & 0.993 & & 0.990 & 0.000 & 0.000 & 0.003 \\
250 & 0.012 & & 0.008 & 0.000 & 0.000 & 0.004 \\
\hline \hline
\end{tabular}

continuously and rapidly rotated. An Au NPs layer was deposited at a deposition angle of $83.4^{\circ}$ on the SCL. The average thickness of the deposited Au was $\sim 10 \mathrm{~nm}$. The optical reflection measurements on the fabricated samples at an incidence angle of $2^{\circ}$ were carried out using a single-beam spectrophotometer with a wavelength range of $300 \mathrm{~nm}<\lambda$ $<1700 \mathrm{~nm}$.

The photoacoustic measurements were performed using a photoacoustic cell $(10.0 \mathrm{~mm}$ in diameter and $3.5 \mathrm{~mm}$ height, MTEC Model 300) on the local plasmon resonators, as well as on a Si wafer and bulk graphite for comparison. We loaded the sample, which was cut into a chip with dimensions $4 \mathrm{~mm} \times 4 \mathrm{~mm} \times 0.5 \mathrm{~mm}$, onto the photoacoustic cell under ambient conditions. The chip was then irradiated with a laser (wavelength $785 \mathrm{~nm}$ ), whose intensity was modulated sinusoidally, from the surface normal. The photoacoustic signal, which was detected by a microphone, was measured with a lock-in amplifier and recorded as a function of the laser modulation frequency over the range from $1 \mathrm{kHz}$ to $100 \mathrm{kHz}$.

\section{RESULTS AND DISCUSSION}

Figure 4 shows the photoacoustic spectra of the local plasmon resonators with $A=0.50 \quad\left(d_{p c l}=20 \mathrm{~nm}\right)$ and $A=0.97\left(d_{p c l}=80 \mathrm{~nm}\right)$, graphite $(A=0.85)$, and Si wafer $(A=0.67)$. These spectra have many distinct peaks at identical positions. The peaks originate from the acoustic resonance in the photoacoustic cell. Apart from these resonance peaks, we focused our attention on the relative amplitudes of the obtained spectra, which qualitatively describe the surface temperature modulation of the samples. The photoacoustic amplitude from the sample with $A=0.50$ is 8 times larger than that of the Si wafer $(A=0.67)$ which has higher optical absorption than the sample. Additionally, the sample with $A=0.97$ generates a photoacoustic amplitude 15 times larger than that of the Si wafer. These results indicate that the localized heat generation of the Au NPs layer, which is much thinner than the photothermally converted layer of a Si wafer ( $\sim 9 \mu \mathrm{m}$ thick), contributes to the efficient photoacoustic 


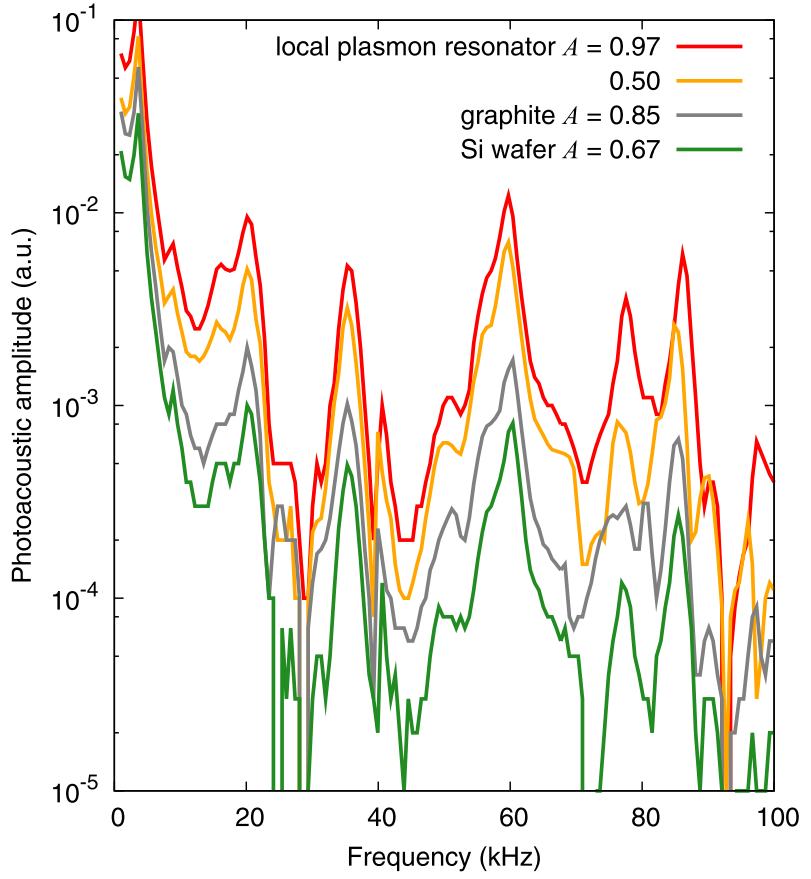

FIG. 4. Photoacoustic spectra of local plasmon resonators with $A=0.50$ and $A=0.97$, graphite $(A=0.85)$, and $\mathrm{Si}$ wafer $(A=0.67)$. The photoacoustic measurements were performed with a $785 \mathrm{~nm}$ wavelength laser.

emission. The photoacoustic amplitude from the sample with $A=0.97$ is 8 times larger than that of the graphite $(A=0.85)$. Consequently, highly localized heat generation in the $\mathrm{Au}$ NPs enables rapid temperature-gradient modulation in the vicinity of the local plasmon resonator surface.

\section{CONCLUSION}

In this report, we investigated the depth profile of optical absorption in the local plasmon resonators. The transfer matrix and energy balance calculations revealed that the light absorption of the local plasmon resonators is highly localized (more than 99\%) in the Au NPs layer. This result suggests that the heat generation is also localized in the thin Au NPs layer $(\sim 10 \mathrm{~nm})$ and can rapidly increase the local temperature. Thus, photoacoustic measurements were performed on the fabricated local plasmon resonators. The photoacoustic amplitude of the local plasmon resonator with a high optical absorption $(A=0.97)$ was 15 times larger than that of the bulk $\mathrm{Si}$ wafer $(A=0.67)$, and 8 times larger than that of graphite $(A=0.85)$. Local plasmon resonators are desirable as nano-heaters which can control local temperatures with a high degree of sensitivity.

\section{ACKNOWLEDGMENTS}

We are grateful to Sadamu Kinoshita from Kyoto University for assisting us with the SEM observations. This work was supported by JSPS KAKENHI Grant Nos. 25286037 and $24 \cdot 2362$.

${ }^{1}$ X. Huang, I. H. El-Sayed, W. Qian, and M. A. El-Sayed, J. Am. Chem. Soc. 128(6), 2115-2120 (2006).

${ }^{2}$ X. Qian, X.-H. Peng, D. O. Ansari, Q. Yin-Goen, G. Z. Chen, D. M. Shin, L. Yang, A. N. Young, M. D. Wang, and S. Nie, Nat. Biotechnol. 26(1), 83-90 (2008).

${ }^{3}$ P. Christopher, H. Xin, and S. Linic, Nat. Chem. 3(6), 467-472 (2011).

${ }^{4}$ O. Neumann, A. S. Urban, J. Day, S. Lal, P. Nordlander, and N. J. Halas, ACS Nano 7(1), 42-49 (2013).

${ }^{5}$ Z. Fang, Y.-R. Zhen, O. Neumann, A. Polman, F. J. G. de Abajo, P. Nordlander, and N. J. Halas, Nano Lett. 13(4), 1736-1742 (2013).

${ }^{6}$ G. L. Liu, J. Kim, Y. Lu, and L. P. Lee, Nat. Mater. 5(1), 27-32 (2006).

${ }^{7}$ J. S. Donner, G. Baffou, D. McCloskey, and R. Quidant, ACS Nano 5(7), 5457-5462 (2011).

${ }^{8}$ D. A. Boyd, J. R. Adleman, D. G. Goodwin, and D. Psaltis, Anal. Chem. 80(7), 2452-2456 (2008).

${ }^{9}$ Y. Hou, J.-S. Kim, M. O’Donnel, and L. J. Guo, Appl. Phys. Lett. 89, 093901 (2006).

${ }^{10}$ V. P. Zharov, Nat. Photon. 5(2), 110-116 (2011).

${ }^{11}$ W. Gotschy, K. Vonmetz, A. Leitner, and F. R. Aussenegg, Opt. Lett. 21(15), 1099-1101 (1996)

${ }^{12}$ W. Hasan, C. L. Stender, M. H. Lee, C. L. Nehl, J. Lee, and T. W. Odom, Nano Lett. 9(4), 1555-1558 (2009).

${ }^{13}$ Y. S. Chen, W. Frey, S. Kim, P. Kruizinga, K. Homan, and S. Emelianov, Nano Lett. 11(2) 348-354 (2011).

${ }^{14}$ K. Namura, M. Suzuki, K. Nakajima, and K. Kimura, Opt. Lett. 36, 3533-3535 (2011).

${ }^{15}$ I. J. Hodgkinson and Q. Wu, Birefringent Thin Films and Polarizing Elements (World Scientific, Singapore 1997).

${ }^{16}$ P. B. Johnson and R. W. Christy, Phys. Rev. B 6(12), 4370-4379 (1972).

${ }^{17}$ L. Shao, K. C. Woo, H. Chen, Z. Jin, J. Wang, and H.-Q. Lin, ACS Nano 4(6), 3053-3062 (2010).

${ }^{18}$ Handbook of Chemistry and Physics (70 ed.), edited by D. R. Lide (Boca Raton, CRC Press, 1990), pp. E-401.

${ }^{19}$ A. Rosencwaig and A. Gersho, J. Appl. Phys. 47(1), 64-69 (1976).

${ }^{20}$ M. Suzuki, Y. Imai, H. Tokunaga, K. Nakajima, K. Kimura, T. Fukuoka, and Y. Mori, J. Nanophotonics 3, 031502 (2009). 